\title{
ON A REPRESENTATION OF THE SOLUTION TO THE DIRICHLET PROBLEM IN A DISK. THE POISSON INTEGRAL BASED SOLUTION IN POLYNOMIALS
}

\author{
V.L. Borsch, I. E. Platonova ${ }^{\dagger}$
}

Communicated by Prof. P. I. Kogut

\begin{abstract}
The representation $u(\boldsymbol{x})=F_{2}(\boldsymbol{x}) Q_{m-2}(\boldsymbol{x})+Q_{m}(\boldsymbol{x})$ for the solution to the Dirichlet problem for the Laplace equation in a disk: $F_{2}(\boldsymbol{x})=\left|\boldsymbol{x}-\boldsymbol{x}_{0}\right|^{2}-c^{2} \leqslant 0$, is proved using the Poisson integral; $Q_{m}(\boldsymbol{x})$ being the polynomial boundary function of degree $m$, $Q_{m-2}(\boldsymbol{x})$ being the uniquely determined polynomial of degree $m-2$.
\end{abstract}

Key words: the Dirichlet problem, the Poisson integral.

2010 Mathematics Subject Classification: 31A25, 31B20, 35A09, 35G15, 35J25.

\section{Introduction}

Consider the well known Dirichlet problem for the Laplace equation in a disk of radius $c$ centered at point $\boldsymbol{x}_{0}$ in the plane $\mathbb{R}^{2}$ parameterized by the cartesian orthogonal coordinates $\boldsymbol{x}=\left(x_{1}, x_{2}\right)$

$$
\left\{\begin{aligned}
\Delta u(\boldsymbol{x}) & =0, & & \boldsymbol{x} \in \mathcal{B}_{c}^{2}\left(\boldsymbol{x}_{0}\right):=\left\{\boldsymbol{x}:\left|\boldsymbol{x}-\boldsymbol{x}_{0}\right|^{2}<c^{2}\right\}, \\
u(\boldsymbol{x}) & =u_{0}(\boldsymbol{x}), & & \boldsymbol{x} \in \mathcal{S}_{c}^{2}\left(\boldsymbol{x}_{0}\right):=\left\{\boldsymbol{x}:\left|\boldsymbol{x}-\boldsymbol{x}_{0}\right|^{2}=c^{2}\right\},
\end{aligned}\right.
$$

where the boundary function $u_{0}(\boldsymbol{x}) \in \mathscr{P} \mathscr{C}\left(\mathcal{S}_{c}^{2}\left(\boldsymbol{x}_{0}\right)\right)$.

The unique solution $u(\boldsymbol{x}) \in \mathscr{C}^{a}\left(\mathcal{B}_{c}^{2}\left(\boldsymbol{x}_{0}\right)\right) \bigcap \mathscr{P} \mathscr{C}\left(\overline{\mathcal{B}_{c}^{2}\left(\boldsymbol{x}_{0}\right)}\right)$ to the problem is known to have some representations [3], for example, $a)$ as the trigonometric series

$$
\stackrel{u}{u}(r, \varphi)=\frac{a_{0}}{2}+\sum_{\mu=1}^{\infty}\left(\frac{r}{c}\right)^{\mu}\left(a_{\mu} \cos (\mu \varphi)+b_{\mu} \cos (\mu \varphi)\right),
$$

where the circle over the function name indicates changing the cartesian coordinates to the polar ones: $x_{1}=x_{1,0}+r \cos \varphi, x_{2}=x_{2,0}+r \sin \varphi,(r, \varphi) \in \overline{\mathcal{B}_{c}^{2}\left(\boldsymbol{x}_{0}\right)} ; a_{0}$, $a_{\mu}$, and $b_{\mu}$ are the Fourier coefficients for $\left.\stackrel{\varkappa}{0}_{0}(\varphi) ; b\right)$ as the Poisson integral

$$
\stackrel{\imath}{u}(r, \varphi)=\frac{1}{2 \pi} \int_{0}^{2 \pi} \frac{\check{u}_{0}(\theta)\left(c^{2}-r^{2}\right) \mathrm{d} \theta}{c^{2}-2 c r \cos (\theta-\varphi)+r^{2}}
$$

\footnotetext{
${ }^{*}$ Dept. of Differential Equations, Faculty of Mech \& Math, Oles Honchar Dnipro National University, 72, Gagarin av., Dnipro, 49010, Ukraine, bv1@dsu.dp.ua

${ }^{\dagger}$ College of Radioelectronics, 18, Schmidt st., Dnipro, 49006, Ukraine, irina.platonoff@mail.ru

(C) V. L. Borsch, I. E. Platonova, 2018.
} 
being the convolution of the boundary function and the Poisson kernel, or $c$ ) as the real part of the Cauchy integral.

But what happens to the solution to the Dirichlet problem (1.1) when the boundary function is a polynomial

$$
u_{0}(\boldsymbol{x})=Q_{m}(\boldsymbol{x})=\sum_{p+q=0}^{m} a_{p, q} x_{1}^{p} x_{2}^{q}=\left\{\left(x_{1}, x_{2}\right) \rightarrow(c, \varphi)\right\}=\stackrel{\circ}{0}_{0}(\varphi),
$$

where $p, q \in \mathbb{Z} \backslash \mathbb{Z}_{-}, a_{p, q} \in \mathbb{R}$ ? It is a very simple question to be answered quickly. The solution is of course a polynomial of the same degree $m$ as the boundary function $Q_{m}(\boldsymbol{x})(1.4)$. But has the polynomial solution a morphology suitable for checking the solution to be valid? The question had puzzled us in academic year 2012-2013 we started as the lecturer and the instructor in the partial differential equations course at the Faculty of Mech \& Math of DNU. Setting up the tutorial Dirichlet problems in polynomials we tried to compose the solution manual in such a way to check the solutions to the problems not pointwise but functionally. The linear boundary functions are exactly the solutions to the problems provided the domains of definition of the boundary functions are extended form $\mathcal{S}_{c}^{2}\left(\boldsymbol{x}_{0}\right)$ to $\overline{\mathcal{B}_{c}^{2}\left(\boldsymbol{x}_{0}\right)}$. The quadratic boundary functions $Q_{2}(\boldsymbol{x})$ lead to the quadratic solutions and are easily represented as follows

$$
u(\boldsymbol{x})=F_{2}(\boldsymbol{x}) b_{0}+Q_{2}^{\prime}(\boldsymbol{x}),
$$

where $b_{0}$ are the uniquely determined constants, and the prime over the boundary functions is explained below in the formulation of the proposition 1.1.

But what about the solution to the Dirichlet problem when the boundary function is a polynomial of the degree higher than second? We had thoroughly studied all the known to us textbooks and solution manuals on the subject in Russian and English but in vain. We had been amazed that no one of the above textbooks or solution manuals answers the question. Therefore, we had to conjecture that the morphology of the solution remains the same as that given by the formula (1.5) where the constant $b_{0}$ is replaced with a polynomial $P_{m-2}(\boldsymbol{x})$ of the order $m-2$. The conjecture had been formulated in [2] as the following

Proposition 1.1. Solution to the Dirichlet problem (1.1), where the boundary function is a polynomial $Q_{m}(\boldsymbol{x})(1.4)$, admits the following representation

$$
u(\boldsymbol{x})=F_{2}(\boldsymbol{x}) Q_{m-2}(\boldsymbol{x})+Q_{m}^{\prime}(\boldsymbol{x}),
$$

where the polynomial $F_{2}(\boldsymbol{x})$ of second degree specifies the boundary of the disk: $F_{2}(\boldsymbol{x})=\left|\boldsymbol{x}-\boldsymbol{x}_{0}\right|^{2}-c^{2}=0, Q_{m-2}(\boldsymbol{x})$ is the uniquely determined polynomial of degree $m-2$, and $Q_{m}^{\prime}(\boldsymbol{x})$ is the evident extension of the boundary function $Q_{m}(\boldsymbol{x})$ from $\mathcal{S}_{c}^{2}\left(\boldsymbol{x}_{0}\right)$ to $\overline{\mathcal{B}_{c}^{2}\left(\boldsymbol{x}_{0}\right)}$ (this means that $c$ is replaced with $r \in[0, c]$ in (1.4)). 
Applying some environments allowing symbolic algebraic manipulations we had successfully tested the conjecture using a lot of the boundary functions, including those of very high degree. Finally, we had proved the proposition using the direct approach based on the trigonometric series representation (1.2) for the solution to the Dirichlet problem and published the proof in [2].

Then we had succeeded in proving the proposition in quite different ways, say, applying the symmetry methods $[1,5,6]$. But when proving the above proposition we had turned out to be involved in the problem of finding the morphology of the Neumann problem in a disk. We had tried to find the representation formula to the Neumann problem posed in polynomials and had found that the integral formula for the solution known in $\mathbb{R}^{2}$ as the Dini integral suits well for this. So, completing our exercises with the Dirichlet problem in a disk posed in polynomials we'd like to present a proof of the above statement fully based on the Poisson integral for the Dirichlet problem.

\section{Proving the representation}

Proof. Firstly, we expand the Poisson kernel into the series [3]

$$
\begin{aligned}
P(\theta ; r, \varphi) & =\frac{c^{2}-r^{2}}{c^{2}-2 c r \cos \theta+r^{2}} \\
& =\frac{1-\varrho^{2}}{1-2 \varrho \cos \theta+\varrho^{2}}=\left\{z=\varrho e^{i \theta}\right\} \\
& =-1+\frac{1}{1-z}+\frac{1}{1-\bar{z}}=-1+\sum_{\gamma=0}^{\infty} z^{\gamma}+\sum_{\gamma=0}^{\infty} \bar{z}^{\gamma} \\
& =1+2 \sum_{\gamma=1}^{\infty} \varrho^{\gamma} \cos (\gamma \theta)=1+2 \sum_{\gamma=1}^{\infty}\left(\frac{r}{c}\right)^{\gamma} \cos (\gamma \theta),
\end{aligned}
$$

absolutely and uniformly convergent in the disk $\mathcal{B}_{c}^{2}\left(\boldsymbol{x}_{0}\right)$ and rewrite the Poisson integral (1.3) as follows

$$
\stackrel{\imath}{u}(r, \varphi)=\frac{1}{2 \pi} \int_{0}^{2 \pi} \stackrel{\circ}{u}_{0}(\theta) P(\theta-\varphi ; r, \varphi) \mathrm{d} \theta=I_{0}(\varphi)+2 \sum_{\gamma=1}^{\infty}\left(\frac{r}{c}\right)^{\gamma} I_{\gamma}(\varphi),
$$

where the integral terms are

$$
I_{\gamma}(\varphi)=\frac{1}{2 \pi} \int_{0}^{2 \pi} \stackrel{\circ}{u}_{0}(\theta) \cos [\gamma(\theta-\varphi)] \mathrm{d} \theta, \quad \gamma \in \mathbb{Z} \backslash \mathbb{Z}_{-} .
$$

Secondly, we consider the monomials $x_{1}^{p} x_{2}^{q} \subseteq Q_{m}(\boldsymbol{x})=u_{0}(\boldsymbol{x})$ (1.4), where $2 \leqslant p+q \leqslant m$, separately, accounting for the following cases to be possible: 1) $p+q$ is an odd: a) $p$ is an odd, $q=0$; b) $p=0, q$ is an odd; c) $p$ is an odd, $q$ is 
an even; d) $p$ is an even, $q$ is an odd; 2) $p+q$ is an even: a) $p$ is an even, $q=0$; b) $p=0, q$ is an even; c) $p$ is an odd, $q$ is an odd; d) $p$ is an even, $q$ is an even, and for the sake of brevity assume that $\boldsymbol{x}_{0}=\mathbf{0}$.

Let $p$ is an odd: $p \geqslant 3$, and $q=0$, then [4]

$$
u_{0}(\boldsymbol{x})=x_{1}^{p}=c^{p} \cos ^{p} \varphi=\frac{c^{p}}{2^{p-1}} \sum_{\mu=0}^{\frac{p-1}{2}} C_{p}^{\mu} \cos [(p-2 \mu) \varphi]=\stackrel{\circ}{u}_{0}(\varphi)
$$

and we calculate the integral terms $(2.2)$ to be

$$
\begin{aligned}
I_{0}(\varphi) & =\frac{1}{4 \pi} \frac{c^{p}}{2^{p-1}} \sum_{\mu=0}^{\frac{p-1}{2}} C_{p}^{\mu} \int_{0}^{2 \pi} \cos [(p-2 \mu) \theta] \mathrm{d} \theta=0, \\
I_{\gamma}(\varphi) & =\frac{1}{4 \pi} \frac{c^{p}}{2^{p-1}} \sum_{\mu=0}^{\frac{p-1}{2}} C_{p}^{\mu} \int_{0}^{2 \pi} \cos [(p-2 \mu+\gamma) \theta-\gamma \varphi] \mathrm{d} \theta \\
& +\frac{1}{4 \pi} \frac{c^{p}}{2^{p-1}} \sum_{\mu=0}^{\frac{p-1}{2}} C_{p}^{\mu} \int_{0}^{2 \pi} \cos [(p-2 \mu-\gamma) \theta+\gamma \varphi] \mathrm{d} \theta \\
& =\frac{1}{2} \frac{c^{p}}{2^{p-1}} C_{p}^{\frac{p-\gamma}{2}} \cos (\gamma \varphi), \quad \gamma=p-2 \mu, \quad 2 \mu=0, \ldots, p-1
\end{aligned}
$$

Since $(p-2 \mu) \in \mathbb{N}$, all the integrals of the formula for the term $I_{0}(\varphi)$ vanish. The same is true for all the integrals in the first sum of the formula for the terms $I_{\gamma}(\varphi)$, and for $p-2 \mu-\gamma \in \mathbb{N}$ and the corresponding integrals in the second sum of the formula. But when $\gamma=p-2 \mu$, then $\cos [(p-2 \mu-\gamma) \theta+\gamma \varphi]=$ $\cos (\gamma \varphi)$, and the corresponding integrals in the second sum are equaled to $2 \pi \cos (\gamma \varphi)$.

Thirdly, we substitute the above non-zero integral terms $I_{\gamma}(\varphi)$ into the integral formula (2.1)

$$
\begin{aligned}
\stackrel{i}{u}(r, \varphi) & =\frac{c^{p}}{2^{p-1}} \sum_{\mu=0}^{\frac{p-1}{2}} C_{p}^{\mu}\left(\frac{r}{c}\right)^{\gamma} \cos (\gamma \varphi) \\
& =\frac{r^{p}}{2^{p-1}} \cos (p \varphi)+\frac{1}{2^{p-1}} \sum_{\mu=1}^{\frac{p-1}{2}} C_{p}^{\mu} r^{p-2 \mu} c^{2 \mu} \cos [(p-2 \mu) \varphi]
\end{aligned}
$$

and rearrange the last sum as follows 


$$
\begin{aligned}
& \sum_{\mu=1}^{\frac{p-1}{2}} C_{p}^{\mu} r^{p-2 \mu} c^{2 \mu} \cos [(p-2 \mu) \varphi]=\sum_{\mu=1}^{\frac{p-1}{2}} C_{p}^{\mu} r^{p-2 \mu}\left(c^{2 \mu} \mp r^{2 \mu}\right) \cos [(p-2 \mu) \varphi] \\
= & \sum_{\mu=1}^{\frac{p-1}{2}} C_{p}^{\mu} r^{p} \cos [(p-2 \mu) \varphi]+\sum_{\mu=1}^{\frac{p-1}{2}} C_{p}^{\mu} r^{p-2 \mu}\left(c^{2 \mu}-r^{2 \mu}\right) \cos [(p-2 \mu) \varphi] \\
= & \sum_{\mu=1}^{\frac{p-1}{2}} C_{p}^{\mu} r^{p} \cos [(p-2 \mu) \varphi]-\left(r^{2}-c^{2}\right) \sum_{\mu=1}^{\frac{p-1}{2}} C_{p}^{\mu} r^{p-2 \mu} A_{\mu}(r) \cos [(p-2 \mu) \varphi],
\end{aligned}
$$

where the factorization of the binoms $c^{2 \mu}-r^{2 \mu}$ is used, and

$$
A_{\mu}(r)=\left\{\begin{aligned}
1, & \mu=1, \\
r^{2 \mu-2}+c^{2} r^{2 \mu-4}+\ldots+c^{2 \mu-4} r^{2}+c^{2 \mu-2}, & \mu>1 .
\end{aligned}\right.
$$

Gathering all the terms, we obtain the solution to the Dirichlet problem (1.1)

$$
\begin{aligned}
\grave{u}(r, \varphi) & =\frac{r^{p}}{2^{p-1}} \sum_{\mu=0}^{\frac{p-1}{2}} C_{p}^{\mu} \cos [(p-2 \mu) \varphi] \\
& -\frac{r^{2}-c^{2}}{2^{p-1}} \sum_{\mu=1}^{\frac{p-1}{2}} C_{p}^{\mu} r^{p-2 \mu} A_{\mu}(r) \cos [(p-2 \mu) \varphi] \\
& \stackrel{(2.3)}{=} \stackrel{\circ}{0}_{0}^{\prime}(r, \varphi)+\stackrel{\circ}{F}_{2}(r, \varphi) \stackrel{\circ}{p-2}_{p}(r, \varphi) .
\end{aligned}
$$

The other monomials $x_{1}^{p} x_{2}^{q}$ are treated the same way.

\section{Acknowledgements}

The article had been submitted, Prof. P. I. Kogut pointed us to the article [7]. We would like to express our gratitude to Prof. P.I. Kogut for the reference.

\section{References}

1. G. W. Bluman, S. KumeI, Symmetries and Differential Equations, Springer, New York, 1989. (Applied Mathematical Sciences, Vol 81)

2. V. L. Borsch, I. E. Platonova, On a representation of the solution to the Dirichlet problem in a circle, Visnyk DNU, Series: Mathematical Modelling, Dnepropetrovsk: DNU, 24(9) (2017), 1-18.

3. E. Goursat, Cours d'Analyse Mathématique: Tome 3, Gauthier-Villars, Paris, 1915. (Cours de la Faculté des Sciences de Paris) 
4. I. S. Gradshteyn, I. M. Ryzhik, Table of Integrals, Series, and Products: translated from Russian, Academic Press, Amsterdam, 2007.

5. P. J. Olver, Applications of Lie Groups to Differential Equations, Springer, New York, 1993. (Graduate Texts in Mathematics, Vol 107)

6. L. V. Ovsyannikov, Group Analysis of Differential Equations: translated from Russian, Academic Press, New York, 1982.

7. A.S. SerToz, Dirichlet problem for polynomials on the unit disk, Communications of Korean Mathematical Society, 29(3) (2014), 415-420. 